\title{
Abundance Evolution in Rotating Magnetised Stars
}

\author{
Christopher A. Tout*, Adrian T. Potter \\ Institute of Astronomy, The Observatories, Madingley Road, Cambridge CB3 OHA, England \\ E-mail: cateast.cam.ac.uk
}

and Shashikumar M. Chitre

Centre for Excellence in Basic Sciences, University of Mumbai, Mumbai 400098, India

\begin{abstract}
We implement equations to evolve angular momentum and poloidal and toroidal magnetic field components as a function of radius in the Cambridge STARS evolution code. The implementation is deliberately flexible so that different physical models can be treated with exactly the same background stellar physics. In particular physical processes that tend to either uniform angular rotation or uniform angular momentum within a star can easily be modelled. Importantly feedback of the magnetic field on angular momentum transport is treated as well as the effects of differential rotation on magnetic fields. One test of such models is the reproduction of abundance distribution with surface rotation velocities. Our models are notably able to reproduce the observed distribution, of both rapidly and slowly rotating nitrogen-enriched stars, when both magnetic fields and angular momentum transport are included.
\end{abstract}

XII International Symposium on Nuclei in the Cosmos,

August 5-12, 2012

Cairns, Australia

\footnotetext{
${ }^{*}$ Speaker.
} 


\section{Introduction}

Evolution of rotation in radiative zones of stars is strongly coupled to evolution of magnetic fields. In most models magnetic flux is redistributed by magneto-rotational turbulence which is also responsible for generating of flux by an $\alpha$ dynamo. This is important to maintain poloidal flux while toroidal flux is maintained by conversion of poloidal flux by shear. Observed fields are potentially strong enough to affect both chemical mixing and angular momentum transport and so the evolution of magnetic fields must be included alongside the evolution of angular momentum by purely hydrodynamical turbulence in any full stellar model [1]. Sufficiently strong magnetic fields also lead to magnetic braking of the whole star and so such fields might explain the existence of slowly rotating but chemically peculiar stars [2]. Unlike Spruit [3] who made magnetic field strength purely a function of stellar structure and rotation, in our models we evolve both poloidal and toroidal components of magnetic field according to advection-diffusion equations derived from the induction equation. These are similar to the equations we use to model the evolution of angular momentum and the two sets are coupled by turbulent diffusivities, magnetic stresses and conversion of poloidal to toroidal field by differential rotation and the regeneration of poloidal field by an $\alpha$ effect. For a set of stars we examine how the magnetic fields, rotation and chemical composition at the surface evolve with time.

\section{Surface Compositions}

Figure 1, sometimes referred to as a Hunter diagram [2], shows stars in a plot of surface nitrogen abundance against surface velocity. As hydrogen burning proceeds by the catalytic CNO cycle carbon is converted to nitrogen deep within the star. Rotationally or magnetically driven turbulence can mix some of the excess nitrogen across the intervening radiative zone to the surface of the star. Thus we might expect to see a correlation between surface velocity and nitrogen abundance and indeed we do. However there also exists a class of slowly rotating but equally nitrogen-enhanced stars. Analysis of the two groups shows no apparent other difference in their composition but we note that the mass range $\left(8<M / M_{\odot}<20\right)$ of the whole observed sample is rather small and so we might expect the stars to have rather similar underlying evolution.

When we model a standard population of stars similar to those in the observed sample we find that we do indeed produce both classes of nitrogen-enriched stars. This is because, in our model, only stars less massive than about $15 M_{\odot}$ can sustain a strong surface magnetic field (figure 2). In the more massive stars, diffusion owing to shear and the Kelvin-Helmholtz instability exceeds diffusion owing to magnetic instabilities throughout a sufficiently large region of the radiative envelope. The $\alpha$ effect decays along with the magnetic field and any magnetic field decays exponentially. When the magnetic diffusion dominates an equilibrium can be reached in which the $\alpha$ effect regenerates field as fast as it diffuses away and a global field can be maintained. This is because the alpha effect tends to depend linearly on field strength while magnetic instabilities depend on its square.

All the stars initially have a spread of surface rotation velocities. Stars in the lower-mass, magnetic population spin down rapidly by magnetic braking but the magnetic fields continue to drive turbulent mixing through the radiative zone, thus making them chemically peculiar but slowly 


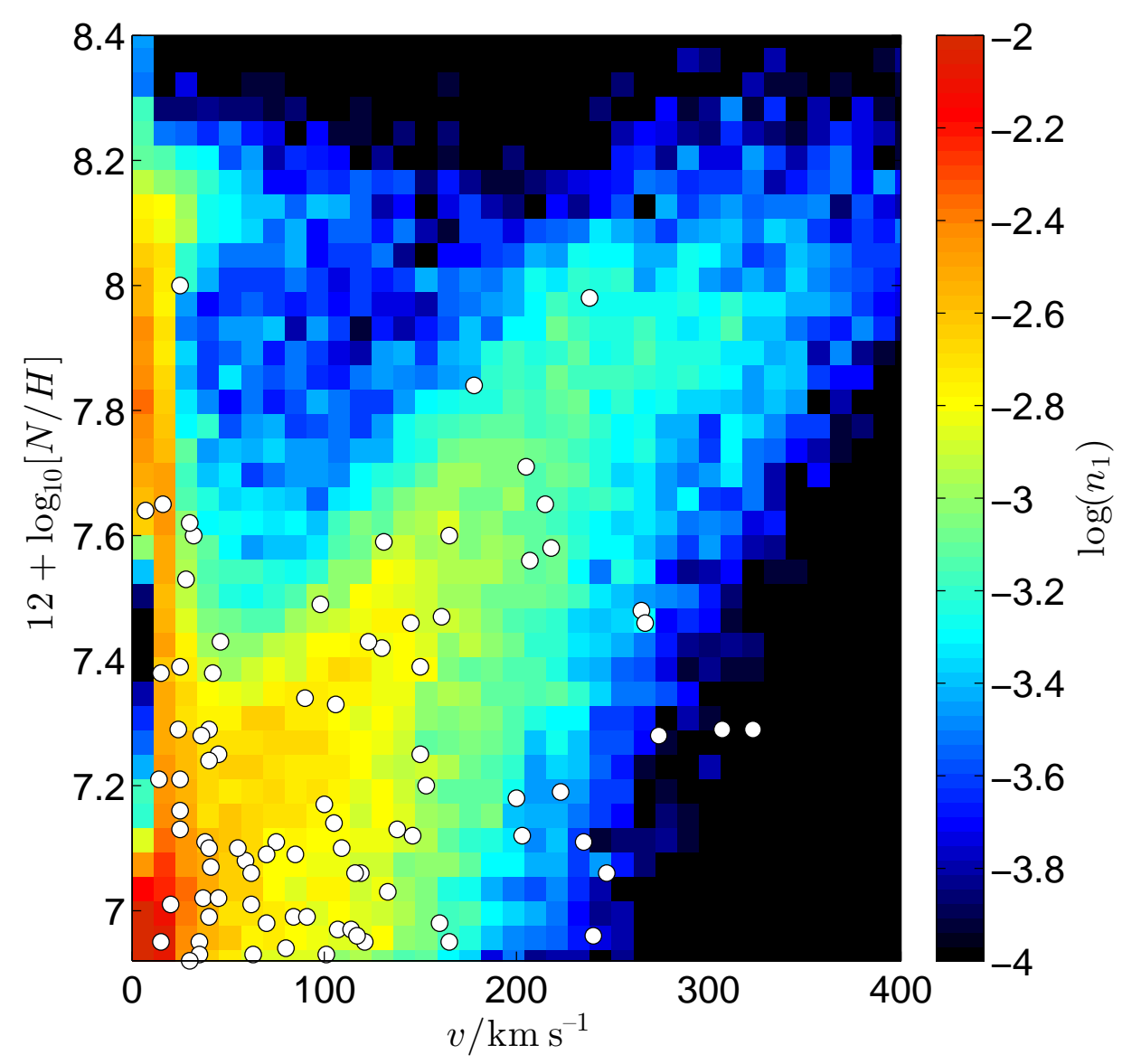

Figure 1: The density of stars in surface nitrogen abundance, surface velocity space for a population of massive stars distributed according to a Salpeter IMF with initial velocities chosen from a Gaussian with mean of $145 \mathrm{~km} \mathrm{~s}^{-1}$ and standard deviation of $94 \mathrm{~km} \mathrm{~s}^{-1}$. The number of stars in each bin divided by the total is $n_{1}$. Points are observed stars in the VLT-FLAMES survey. Our magnetic model reproduces both the stars in which nitrogen abundance is correlated with surface velocity and those that have increased nitrogen but low velocity. More massive stars that, cannot support a dynamo, are enriched by rotational mixing and continue to spin rapidly. Lower-mass stars are spun down rapidly by magnetic braking and are enriched by magnetic mixing.

rotating. The more massive stars evolve just as in non-magnetic models. Hydrodynamic turbulence drives mixing, which is stronger the more rapidly rotating stars, through the radiative zone.

\section{Conclusions}

We have modelled rotating massive stars including an $\alpha-\Omega$ dynamo in radiative zones. In stars greater than about $15 M_{\odot}$ the dynamo cannot be sustained against the effects of hydrodynamic turbulence. Rotationally driven mixing leads to surface nitrogen enrichment which is correlated with surface velocity in these stars. Less massive stars with low rotation rates also evolve in a similar way. 


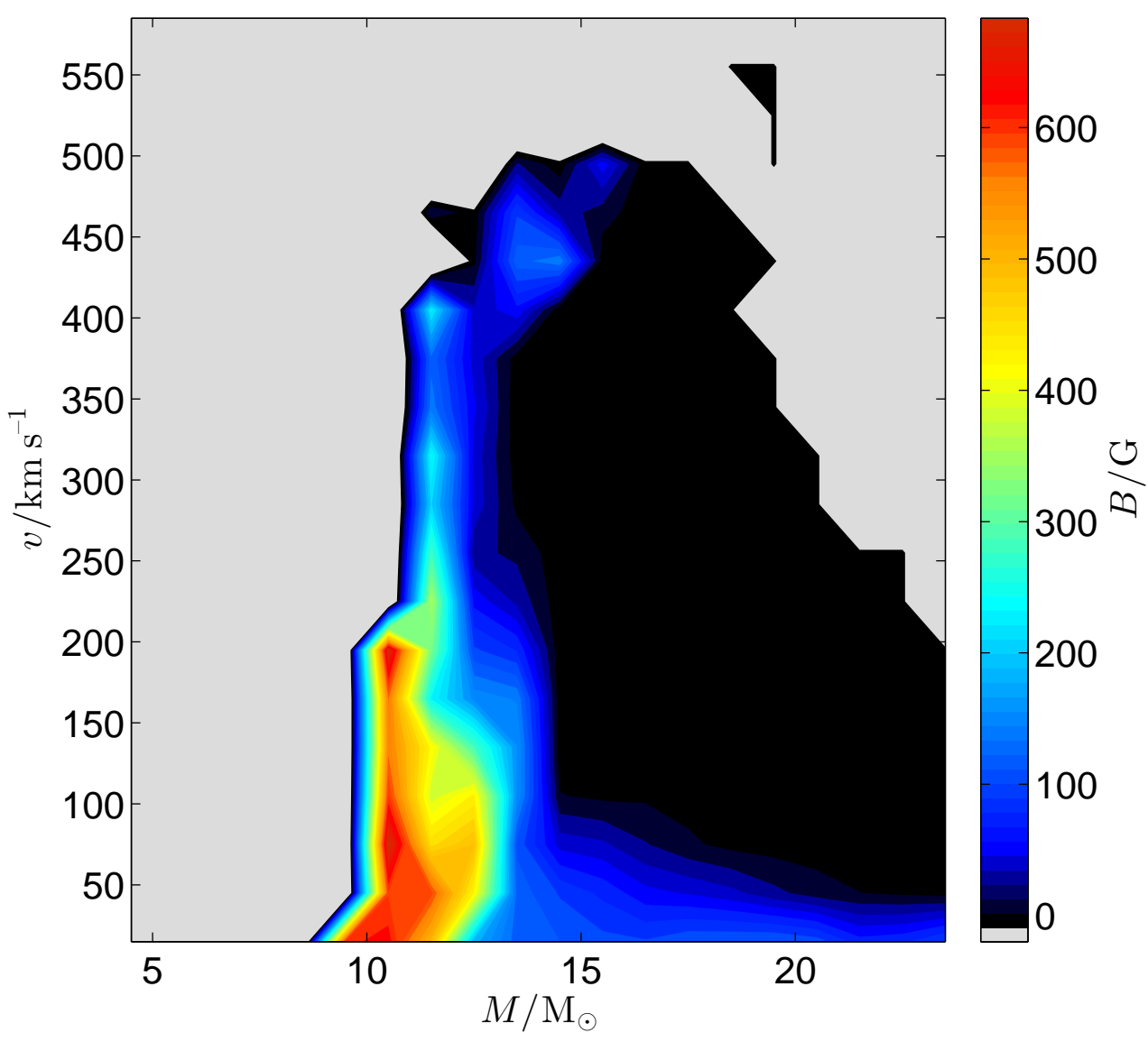

Figure 2: The distribution of magnetic field strengths for the same population of stars used to generate figure 1 by mass and surface rotation velocity. No stars in the observed poulation occupy the grey region. We see that the magnetic stars, which become the slowly rotating but enriched stars exist only in a narrow region around $10 M_{\odot}$. At lower mass they are too faint and at higher mass the field cannot be sustained.

Less massive stars with high rotation rates sustain active dynamos and exhibit strong magnetic fields. They are spun down quickly by magnetic braking but magnetically driven turbulence lasts long enough to mix significant amounts of nitrogen to their surfaces. Again the amount of enhancement is correlated with the initial surface velocity.

If this model is correct examination of these two populations should reveal that members of the slowly rotating, nitrogen-enriched population tend to be more massive and more magnetic. We would also expect the incidence of strong magnetic fields to to fall off in very massive stars.

\section{References}

[1] A. T. Potter, S. M. Chitre and C. A. Tout, 2012, MNRAS, 424, 2358

[2] I. Hunter et al., 2009, A\&A, 496, 841

[3] H. C. Spruit, 2002, A\&A, 381, 923 